\title{
Rutin Improves Bone Histomorphometric Values by Reduction of Osteoclastic Activity in Osteoporosis Mouse Model Induced by Bilateral Ovariectomy
}

\author{
Hye-Hwa Lee, Jae-Won Jang, ${ }^{1,2}$ Jung-Kil Lee, ${ }^{1,3}$ Choon-Keun Park ${ }^{2}$ \\ Research Institute of Medical Sciences,' Chonnam National University, Gwangju, Korea \\ Department of Neurosurgery, Leon Wiltse Memorial Hospital, Suwon, Korea \\ Department of Neurosurgery, ${ }^{3}$ Chonnam National University Hospital, Gwangju, Korea
}

Objective : Osteoporosis is a disease of unbalanced bone metabolism that results in low bone mineral density with increased bone fragility and propensity for fractures. The increased rate of bone fracture due to osteoporosis places a significant burden on public health care expenditures. Therefore, numerous studies have been designed and performed to identify the drugs or health foods that can improve the bone quality or quantity. This study was designed to evaluate and analyze the therapeutic effects of rutin on histomorphometric values of the spine and femur in an osteoporotic mouse model induced by bilateral ovariectomy.

Methods : Thirty female ICR mice (8 weeks old) underwent either a sham operation (only abdominal incision, sham group, $\mathrm{n}=10$ ) or bilateral ovariectomy $(n=20)$. The ovariectomized $(O V X)$ animals were randomly divided into two groups : untreated OVX group (OVX-C, $n=10)$, or rutin-administered group (OVX-R, $n=10)$. The OVX-C group received weight-adjusted doses of saline vehicle and the OVX-R group received $50 \mathrm{mg} / \mathrm{kg}$ of rutin intraperitoneally, starting 1 day after surgery. At 4 and 8 weeks after surgery, serum estrogen, osteocalcin, alkaline phosphatase (ALP), and the telopeptide fragment of type I collagen C-terminus (CTX-1) were analyzed. Interleukin (IL)-1 $\beta$, LL-6, IL-10, and tumor necrosis factor (TNF)- $\alpha$ were also analyzed. Bone histomorphometric parameters of the 4th lumbar vertebra and femur were determined by micro-computed tomography.

Results : In OVX-C group, ALP, osteocalcin, CTX-1, IL-1 $\beta$, IL- 6 , and TNF- $\alpha$ levels were significantly increased at 4 and 8 weeks compared to sham operation group. Rutin administration after OVX statistically significantly reduced ALP, CTX-1, IL-1 13 , IL-6, and TNF- $\alpha$ levels at 4 and 8 weeks. Rutin administration also improves bone histomorphometric parameters including trabecular bone volume fraction, trabecular thickness, and trabecular number. Trabecular separation was also decreased in OVX-R group compared to OVX-C group.

Conclusion : The present study demonstrated that rutin has therapeutic effects on improving bone histomorphometric values in an OVX mouse model. The improvement in histomorphometric values may be associated with the reduction of osteoclastic activity via inhibition of IL-1 $\beta$, IL-6, and TNF- $a$. In future studies, the mechanism for the effect of rutin on osteoporosis should be demonstrated more clearly to use rutin in human osteoporosis.

Key Words : Histomorphometric values · Mice · Osteoclast · Osteoporosis · Rutin.

- Received : April 15, 2019 •Revised : June 9, $2019 \bullet$ Accepted : June 28, 2019

- Address for reprints : Jae-Won Jang

Department of Neurosurgery, Leon Wiltse Memorial Hospital, 437 Gyeongsu-dearo, Paldal-gu, Suwon 16480, Korea

Tel : +82-31-240-6061, Fax : +82-31-223-2322, E-mail : genius60@hanmail.net, ORCID : https://orcid.org/0000-0001-5555-4359

This is an Open Access article distributed under the terms of the Creative Commons Attribution Non-Commercial License (http://creativecommons.org/licenses/by-nc/4.0) which permits unrestricted non-commercial use, distribution, and reproduction in any medium, provided the original work is properly cited. 


\section{INTRODUCTION}

An imbalance between bone formation and resorption due to aging and hormone disruption may lead to the development of osteoporosis ${ }^{10,20)}$. Nowadays, the incidence of osteoporosis is increasing rapidly due to a rise in the aging population. Osteoporosis increases the risk of bone fracture, and osteoporosis-related fractures in an aging population have put a significant strain on the public health $\operatorname{cost}^{2}$. Therefore, prevention and treatment of osteoporosis are hot topics in recent aging society and geriatric medicine.

There are two options for medical treatment of osteoporosis. One option is antiresorptive agents that prevent bone resorption and increase bone density, and the other option is anabolic agents that increases bone formation. The representative drug of antiresorptive agents is bisphosphonates (BPs), and they have been regarded as the first-line drug for the treatment of osteoporosis and are used worldwide ${ }^{7,22)}$. However, it is well-known that BPs are associated with esophageal irritation and they can also aggravate the upper gastrointestinal symptoms ${ }^{21)}$. Moreover, a recent study reported numerous cases of osteonecrosis of the jaw and atypical femoral fractures associated with the long-term use of BPs ${ }^{9,15)}$. These adverse effects on the bone may be due to excessive suppression of bone turnover associated with the long-term use of BPs. Recently, teriparatide (recombinant human parathyroid hormone, rhPTH) has been used as a bone anabolic agent that reduces the risk of vertebral and non-vertebral fractures in severe osteoporotic patients ${ }^{4,24)}$. But, the cost of teriparatide is high; therefore, the use of teriparatide is restricted till now.

Because of the side effect of BPs and the high cost of teriparatide, the need for natural alternatives for prevention and treatment of osteoporosis has greatly increased ${ }^{3,20,28)}$. For the evaluation of the effectiveness and safety of natural products, an experimental osteoporotic animal model is required. Although no single animal model precisely mimics the human condition of osteoporosis, an ovariectomized (OVX) rat or mouse model is the most frequently used model for studying post-menopausal osteoporosis with estrogen deficiency ${ }^{6,23)}$.

Rutin (quercetin-3-rhamnosyl glucoside) is a flavonoid glycoside found in buckwheat which is abundantly present in vegetables, fruits, tea, wine, and herbs. It has several physiological abilities as a good radical scavenger along with antioxidant, anticancer, and anti-inflammatory properties ${ }^{9,16)}$. More- over, bone protective effects of rutin via antioxidation have been reported ${ }^{13)}$. The present study was designed to investigate the effect of rutin in OVX mice via analyses of bone histomorphometric values of the 4th lumbar vertebra and the femur using micro-computed tomographic (CT) scans, and bone turnover markers and cytokines, such as interleukin (IL)-1 $\beta$, IL-6, IL-10, and tumor necrosis factor (TNF)- $\alpha$.

\section{MATERIALS AND METHODS}

All procedures and care were performed in animal research laboratory in Chonnam National University Hospital and study was approved by Chonnam National University Animal Care and Committee.

\section{Experimental animals}

All animal experiments were performed in accordance with the animal care guidelines. A total of 30 female ICR mice (7 weeks old) weighing 30 to $32 \mathrm{~g}$ were maintained in an air-conditioned room (relative humidity $45-65 \%$ ) at $20-22^{\circ} \mathrm{C}$ under a 12-h light/dark cycle and given free access to food and tap water during 1 week.

Each mouse was anesthetized with $2 \%$ isoflurane and maintained with $1 \%$ isoflurane in an oxygen/air mixture by using a gas anesthesia mask mounted on a stereotaxic frame. The mice in the sham group (group $1, n=10$ ) underwent a sham operation (only underwent abdominal incision) at 8 weeks of age. Osteoporosis model was induced by bilateral ovariectomy via a midline abdominal incision. Total 20 animals underwent bilateral ovariectomy and they were randomly divided into two groups. One group (group 3, rutin-administered [OVX-R], n=10) received $50 \mathrm{mg} / \mathrm{kg}$ of rutin intraperitoneally, starting 1 day after ovariectomy, and administration was performed once a day until the time the animal was sacrificed. The other group (group 2, untreated OVX [OVX-C], n=10) received weight-adjusted doses of saline vehicle according to the same schedule. Body weights were checked once a week during the experimental period.

Four weeks after surgery, five mice from each of the three groups were euthanized, and the 4th lumbar vertebra and the femur were removed and blood samples were collected from the retinal artery. Eight weeks after OVX or sham operations, the remaining mice in each group were also euthanized, and 
the 4th lumbar vertebra and the femur were removed and blood samples were collected. Collected blood was centrifuged at $3000 \mathrm{rpm}$ for 10 minutes at $4^{\circ} \mathrm{C}$ to separate the plasma and the serum was stored at $-80^{\circ} \mathrm{C}$. Collected femur and 4 th lumbar vertebra were fixed in $4 \%$ paraformaldehyde for 16 hours, and stored in $80 \%$ ethanol at $4^{\circ} \mathrm{C}$.

\section{Serum biochemical markers of bone metabolism}

Serum estrogen (estradiol, E2) level was determined using a mouse estrogen ELISA kit (MyBioSource, San Diego, CA, USA). Concentrations of serum osteocalcin and alkaline phosphatase (ALP), which are generally regarded as the markers of bone formation, were determined using mouse osteocalcin and ALP ELISA kit (Uscn Life Science Inc., Hubei, China). Serum levels of C-terminal telopeptide fragment of type I collagen C-terminus (CTX-1), which is generated by the osteoclasts and is a marker of bone resorption, was determined using a mouse CTX-1 ELISA kit (Uscn Life Science Inc.).

\section{Multiplex Immunobead Assay}

The levels of interleukin IL-1 $\beta$, IL-6, IL10, and TNF- $\alpha$ in the mouse serum were measured using a multiplex immunobead assay (Luminex 200; Luminex Corp., Austin, TX, USA). The supernatants were added to wells containing the appropriate cytokine bead mixture that included mouse monoclonal antibodies specific for IL-1 $\beta$, IL-6, IL-10, and TNF- $\alpha$ (Panomics, Santa Clara, CA, USA) for 60 minutes. A standard curve was generated by adding dilutions of cytokines to wells. The plate was incubated for 30 minutes at room temperature with the biotinylated detection antibody. After three washes with assay buffer, the reactions were detected by the addition of streptavidin-phycoerythrin using an analysis system (xPONENT, Austin, TX, USA).

\section{Analysis using micro-CT}

Collected femurs and 4 th lumbar vertebrae were cleaned of the soft tissue, and fixed in $80 \%$ ethanol. Bone histomorphometric parameters and the microarchitectural properties of the 4 th lumbar vertebra and the femur were determined using a micro-CT scan (Skyscan, Kontich, Belgium). The X-ray source was set at $50 \mathrm{kV}$ and $200 \mathrm{~A}$, with a $0.5 \mathrm{~mm}$ aluminum filter. Images were captured with a pixel size of $17.09 \mathrm{~m}$, and an angular range of 180 degrees. The exposure time was 1.2 seconds. Raw images were reconstructed into serial cross sec- tion images with identical thresholds for all samples (0 to 6000 in Hounsfield units) by use of the image reconstruction software (CTAN, Kontich, Belgium).

For the bone analysis, bone tissues, the region of the entire 4th lumbar vertebra and of $2-4 \mathrm{~mm}$ from the growth plate of the femur were selected as the region of interest. Image information was obtained based on the automatic domain values produced by the computer. Trabecular bone volume fraction (BV/TV, \%), trabecular thickness (Tb.Th., mm), trabecular number (Tb.N., $1 / \mathrm{mm}^{3}$ ), and trabecular separation (Tb.S., $\mathrm{mm}$ ) were used for the quantitative analysis.

\section{Statistical analysis}

All statistical comparisons were made using SPSS ver. 17.0 (SPSS Inc., Chicago, IL, USA). Data are expressed as means+standard deviations. Repeated measures one-way analysis of variance was used to compare body weights, serum bone turnover markers, cytokines, and bone histomorphometric values between the three groups. One-way analysis of variance was used to identify significant differences between the groups, and $p$ values $<0.05$ were considered statistically significant.

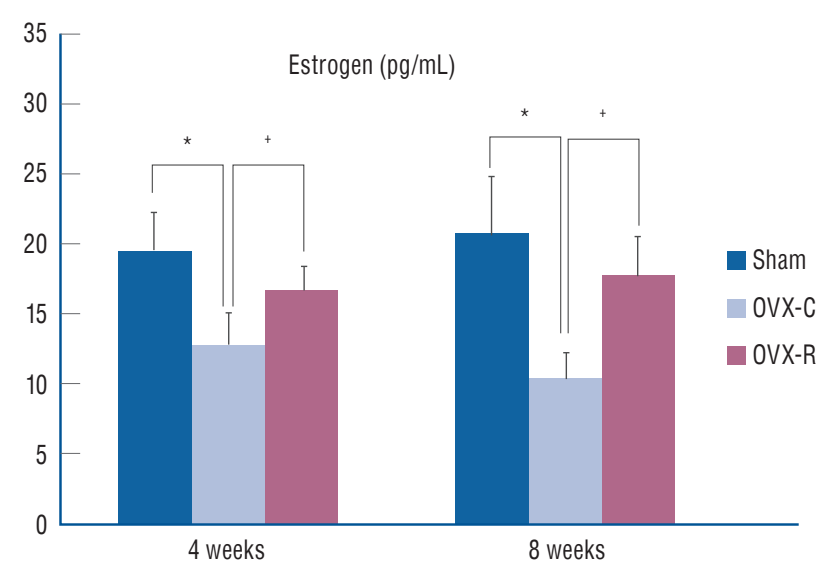

Fig. 1. Graph showing the changes of serum levels of estrogen (estradiol, E2). ${ }^{*} p<0.05$ for the values of OVX-C or OVX-R group compared with that of sham group. ${ }^{\dagger} p<0.05$ for the values of the OVX-C group compared with that of OVX-R group. OVX : ovariectomized, OVX-C : untreated OVX, OVX-R : rutin-administered. 


\section{RESULTS}

\section{Body weights and estrogen levels}

Body weights were checked once a week throughout the 8 -week experimental period. Body weights were similar in the three groups at the start of the study. However, the mean body weight at 4 weeks after surgery in the OVX-C group and OVX-R group was significantly higher than that in the sham group, and this statistical significance was maintained throughout the experimental period ( $p=0.033$ and $p=0.016$, at 4 and 8 weeks, respectively).

As shown in Fig. 1, 4 and 8 weeks after surgery, serum estradiol levels were significantly lower in the OVX-C group compared to the sham and OVX-R group ( $p=0.008$ and $p=0.000$, respectively). However, there was no significant difference in the serum estradiol level between the sham and OVX-R groups, although the levels of estradiol were relatively higher in sham group compared to OVX-R group ( $p=0.187$ ).

\section{Serum biochemical markers of bone turnover}

Fig. 2A and $\mathrm{B}$ demonstrate the serum levels of the biochemical markers of bone formation, osteocalcin and ALP, and Fig. 2C shows the serum CTX-1 levels, which is a sensitive marker of bone resorption. Compared with the sham group, the OVX-C and OVX-R groups had higher osteocalcin levels at 4 weeks, which were statistically significant ( $p=0.003$ and $p=0.016$, respectively). The OVX-C groups had higher ALP levels at 4 weeks and 8 weeks compared with the sham and OVX-R group, which were statistically significant ( $p=0.019$ and $p=0.007$, respectively). The serum osteocalcin was maintained at a high level in the OVX-R group compared to the value in the sham group until 8 weeks $(p=0.004)$. The CTX-1 levels were significantly increased at 4 and 8 weeks after surgery in the OVX-C group compared with the sham and OVX-R groups ( $p=0.000$ and $p=0.007$, respectively); however, there was no significant difference in the CTX-1 level between the sham and OVX-R groups during the experimental period $(p=0.264)$.

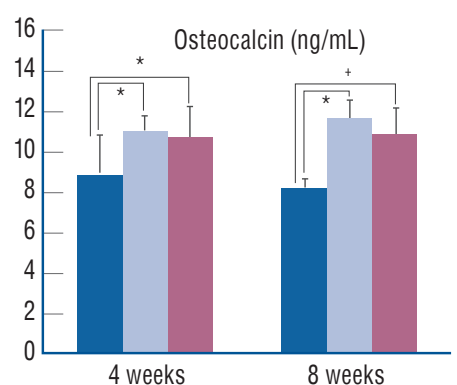

(A)

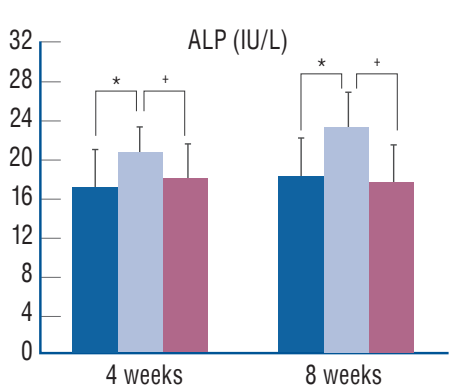

(B)

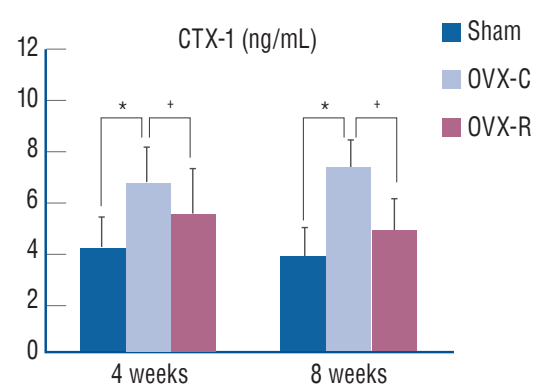

(C)

Fig. 2. Graph showing the changes of serum levels of osteocalcin (A), ALP (B), and CTX-1 (C). ${ }^{*} p<0.05$ for the values of OVX-C or OVX-R group compared with that of sham group. ${ }^{\dagger} p<0.05$ for the values of the OVX-C group compared with that of OVX-R group. OVX : ovariectomized, OVX-C : untreated OVX, OVX-R : rutin-administered, ALP : alkaline phosphatase, CTX-1 : type I collagen C-terminus.

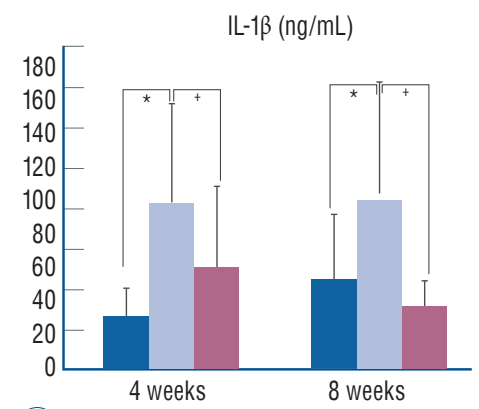

(A)

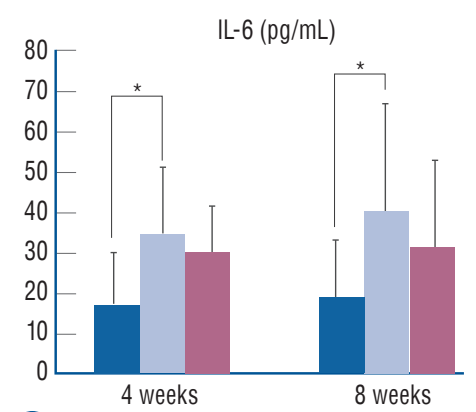

(B)

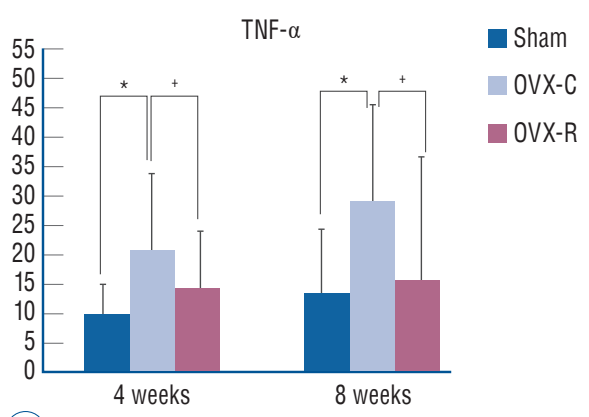

(C)

Fig. 3. Graph showing the changes of serum levels of IL-1 $\beta(A), I L-6(B)$, and TNF- $\alpha(C)$. ${ }^{*} p<0.05$ for the values of OVX-C or OVX-R group compared with that of sham group. ${ }^{\dagger} p<0.05$ for the values of the OVX-C group compared with that of OVX-R group. OVX : ovariectomized, OVX-C : untreated OVX, OVX-R : rutin-administered, IL : interleukin, TNF : tumor necrosis factor. 


\section{Multiplex immunobead assay}

The concentrations of IL- $1 \beta$, IL- 6 , and TNF- $\alpha$ in the mouse serum were significantly increased in the OVX-C group compared to the sham group during the experimental period ( $p=0.000, p=0.022$, and $p=0.017$, respectively); however, the concentration of IL-10 was not detected in the mouse serum during the experimental period (Fig. 3). In the OVX-R group, the concentrations of IL-1 $\beta$ was significantly lower at 4 and 8 weeks after OVX than those in the OVX-C group $(p=0.010$ and $p=0.000$, respectively). TNF- $\alpha$ was also significantly lower at 4 and 8 weeks in the OVX-R group than those in the OVXC group ( $p=0.029$ and $p=0.005$, respectively). The concentration of IL-6 at 4 and 8 weeks after OVX was low in the OVX-R group compared to the OVX-C group; however, there was no statistically significant difference $(p=0.193$ and $p=0.084$, respectively).

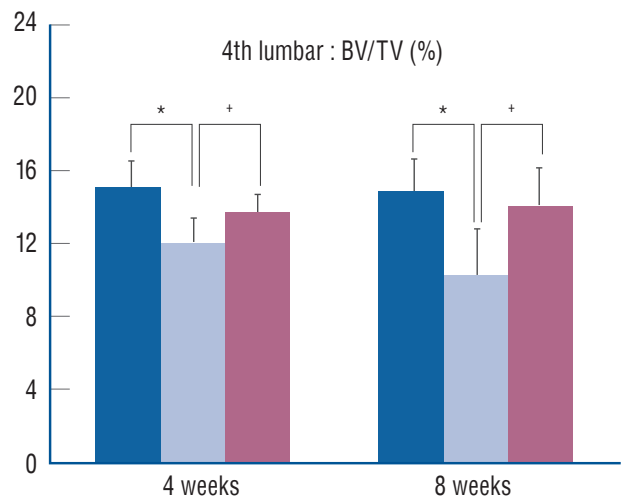

(A)

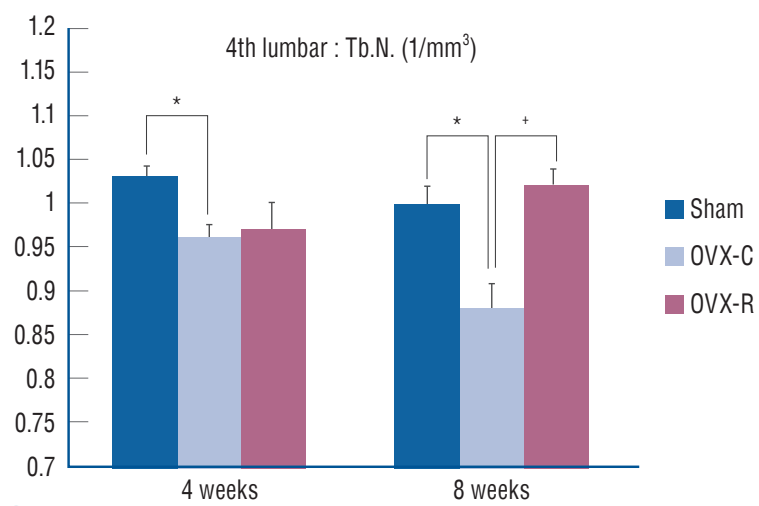

(C)

Fig. 4. Bar graph showing the result of histomorphometric analyses of 4 th lumbar vertebrae in the sham, OVX-C and OVX-R group : BV/TV (A), Tb.Th. (B), Tb.N. (C), and Tb.S. (D). * $p<0.05$ for the values of OVX-C or OVX-R group compared with that of sham group. ${ }^{\dagger} p<0.05$ for the values of the OVX-C group compared with that of OVX-R group. OVX : ovariectomized, OVX-C : untreated OVX, OVX-R : rutin-administered, BV/TV : trabecular bone volume fraction, Tb.Th. : trabecular thickness, Tb.N. : trabecular number, Tb.S. : trabecular separation.

\section{Bone histomorphometric analysis using micro- CT scan}

The values of BV/TV, Tb.Th., Tb.S., and Tb.N. of the femur and 4th lumbar vertebra were measured. BV/TV, Tb.Th., and Tb.N. of the femur and 4 th lumbar vertebra were decreased at 4 and 8 weeks after surgery in the OVX-C group compared to the sham group $(p<0.05$ at 4 and 8 weeks, respectively). Among them, BV/TV and Tb.Th. of the 4th lumbar vertebra were significantly increased at 4 weeks in the OVX-R group compared to the OVX-C group ( $p=0.034$ and $p=0.011$, respectively). Tb.N. was mildly increased at 4 weeks after surgery in the OVX-R group, but there was no significant difference between the OVX-R and OVX-C groups $(p=0.078)$. However, Tb.N. was significantly increased at 8 weeks after surgery in the OVX-R group compared to the OVX-C group ( $p=0.000)$. In contrast, Tb.S. was significantly increased at 4 and 8 weeks

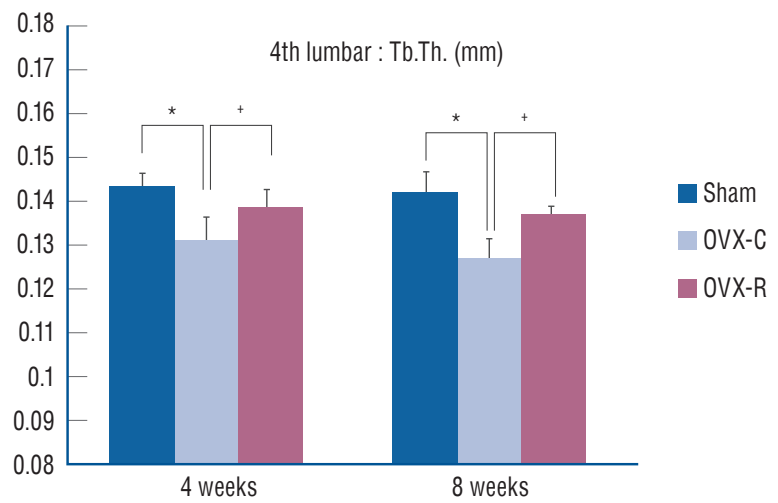

B

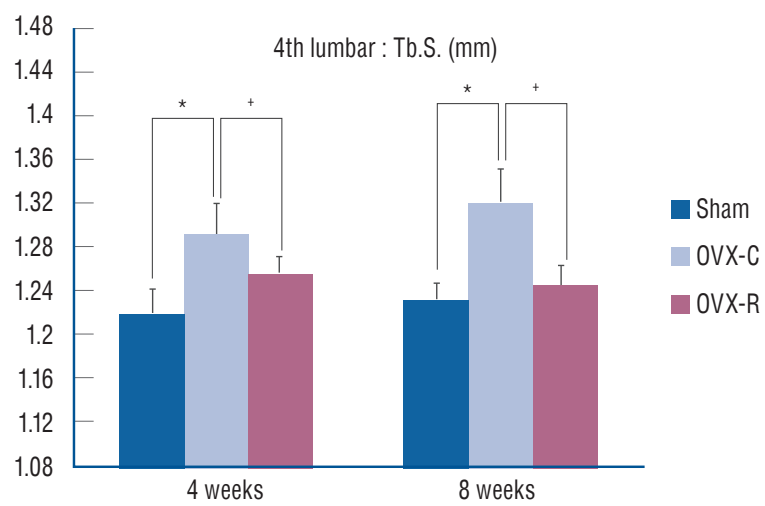

D 


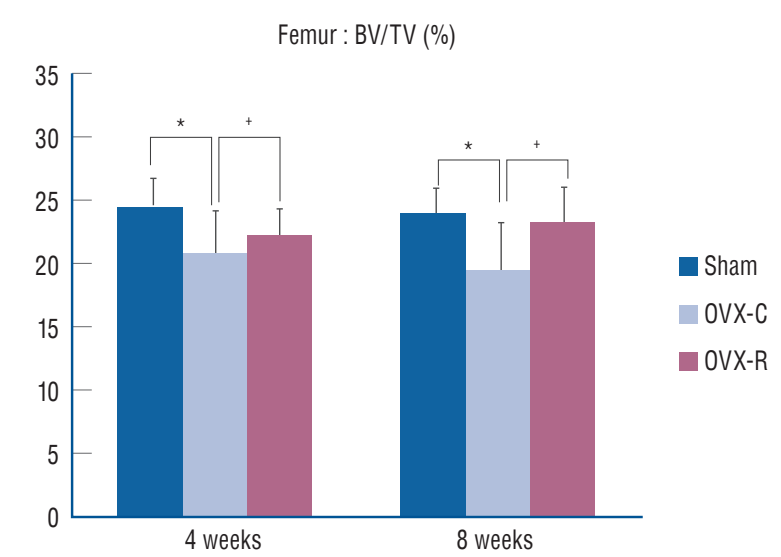

(A)

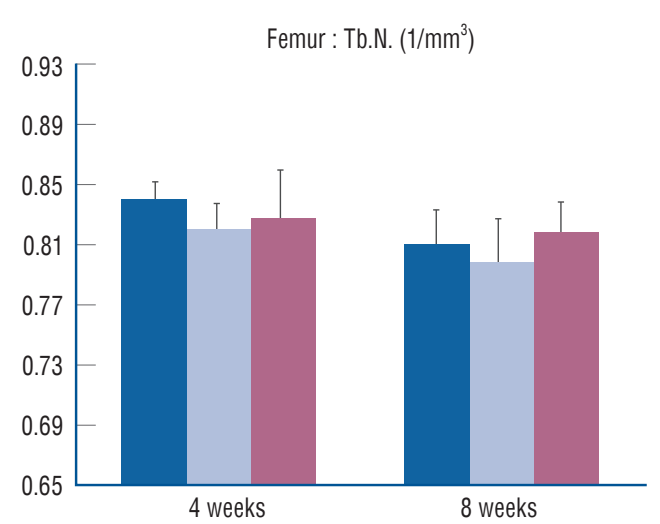

(C)

Fig. 5. Bar graph showing the result of histomorphometric analyses of femur in the sham, OVX-C and OVX-R group : BV/TV (A), Tb.Th. (B), Tb.N. (C), and Tb.S. (D). * $p<0.05$ for the values of OVX-C or OVX-R group compared with that of sham group. ${ }^{\dagger} p<0.05$ for the values of the OVX-C group compared with that of OVX-R group. OVX : ovariectomized, OVX-C : untreated OVX, OVX-R : rutin-administered, BV/TV : trabecular bone volume fraction, Tb.Th. : trabecular thickness, Tb.N. : trabecular number, Tb.S. : trabecular separation.

after surgery in the OVX-C group compared to the sham group, but this value was significantly decreased in the OVX$\mathrm{R}$ group compared to the OVX-C group during the experimental period ( $p<0.05$ at 4 and 8 weeks, respectively) (Fig. 4). In the femur, BV/TV and Tb.Th. were significantly increased at 4 and 8 weeks after OVX in the OVX-R group compared to the OVX-C group ( $p<0.05$ at 4 and 8 weeks, respectively). However, a non-significant increase in Tb.N. was observed in the OVX-R group during the experimental period $(p=0.176)$ (Fig. 5).

The representative micro-CT images of the 4th lumbar vertebra (Fig. 6) after surgery showed that the trabecular bone structure was less in the OVX-C mice compared to the sham mice, and the trabecular bone structure seemed to increase in the OVX-R mice compared to the OVX-C mice.

\section{DISCUSSION}

The impact of osteoporosis is increasingly being recognized in the elderly population, especially in postmenopausal wom$\mathrm{en}^{1,20)}$. One of the primary adverse manifestations of osteoporosis is major skeletal fractures, such as femur or vertebral compression fractures ${ }^{11,27}$. Especially, these fractures can increase the medical cost, and can also be the cause of major morbidity or mortality in elderly patients due to fracture-associated complications ${ }^{2}$. Therefore, adequate treatment of osteopenia or osteoporosis has been a hot topic in geriatric medicine.

BPs, which are analogues of pyrophosphate compounds and inhibit osteoclastic activity, have been considered as the firstline drugs for osteoporosis. Some BPs, such as alendronate cause a fifty percent reduction in major hip or spinal com- 
Sham

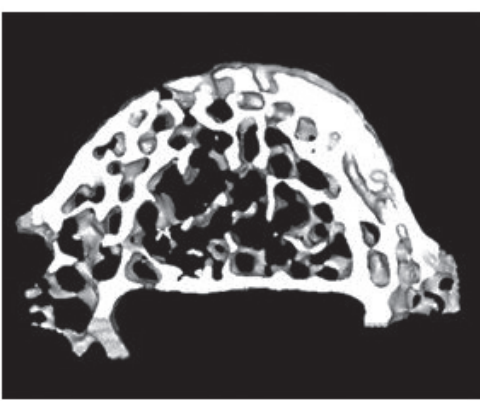

Sham

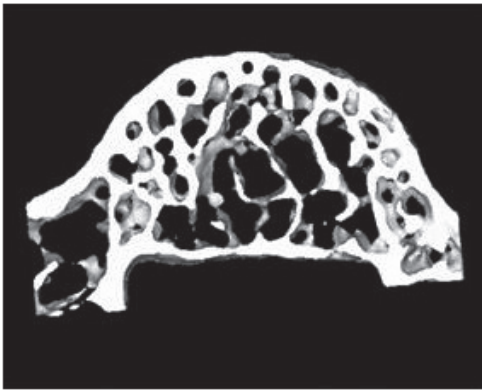

OVX-C

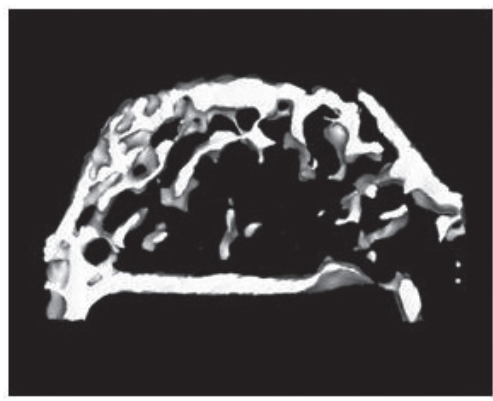

OVX-C

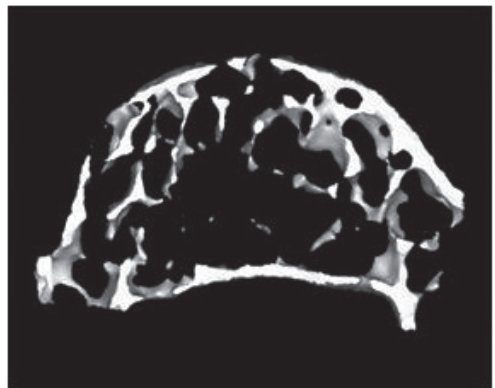

OVX-R

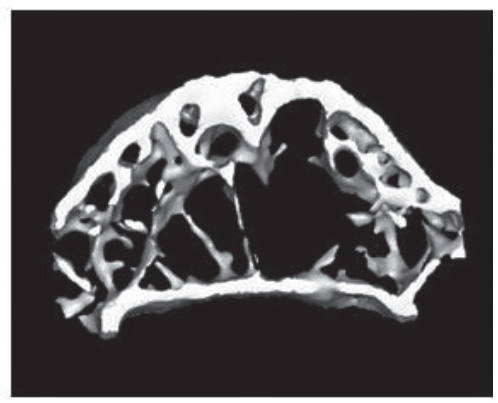

OVX-R

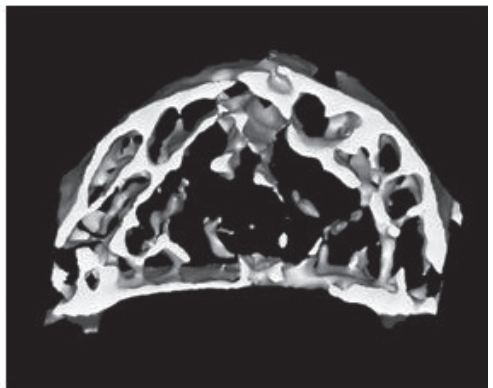

Fig. 6. Representative micro computed tomography (CT) images of the 4th lumbar vertebrae in the sham, OVX-C, and OVX-R groups : 4 weeks (A) and 8 weeks (B) reconstruction images. OVX : ovariectomized, OVX-C : untreated OVX, OVX-R : rutin-administered.

pression fractures with an improvement in BMD scores ${ }^{7)}$. However, there have been many recent reports indicating that there are potentially undesirable side effects related with the long-term usage of BPs, such as atypical femur fractures or osteonecrosis of the jaw ${ }^{3}$. As other pharmacologic agents for osteoporosis, the use of estrogen hormonal replacement therapy has been increasing worldwide, and its effect in increasing the BMD has already been proven ${ }^{26)}$. However, the estrogen hormonal therapy is associated with a significant risk of breast cancer, endometrial cancer, deep vein thrombosis, and cerebrovascular accidents ${ }^{15}$. To reduce the estrogenic effects on breast and endometrial tissue while maintaining the positive effects on bone metabolism, selective estrogen receptor modulators have been developed and used worldwide. However, the problems regarding the risk of breast cancer and stroke still remain controversial ${ }^{19)}$. Recently, rhPTH has been introduced as a bone anabolic agent. This drug has high osteogenic effects in the patients with severe osteoporosis and major skeletal fractures ${ }^{4)}$. However, the drug is expensive in Korea compared to BPs and selective estrogen receptor modulators.

Although many drugs have been developed for the treatment of osteopenia or osteoporosis, there are some limitations, such as cancer and stroke risk, atypical fractures, and high cost ${ }^{4,15,21)}$. Therefore, there is a great need for discovering natural products that have a positive effect on bone health, which can also be easily used as a pharmacologic agent for osteoporosis $^{1,3,5,9)}$.

To investigate the effects of drugs, a successful animal osteoporosis model is firstly required. In the present study, the author used the mouse osteoporosis model induced by bilateral OVX. After bilateral OVX, the serum level of estrogen was decreased, and serum levels of osteocalcin, ALP, and CTX-1 were significantly increased in the OVX-C group compared to the sham group, and this finding indicates increased bone turnover in the OVX-C group. The mean body weight of mice in the OVX-C group was significantly higher than that of mice in the sham group during the experimental period, and the result was comparable to that in the study by Omi and Ezawa ${ }^{18)}$ The body weight gain might be associated with elevated serum osteocalcin level in the OVX-C group. Osteocalcin has a positive effect on lowering the serum glucose level; therefore, an increased osteocalcin level could have decreased excessive serum glucose level, and excessive food intake and weight gain might have occurred in the OVX-C group ${ }^{18)}$. Additionally, the author measured the serum levels of IL-1 $\beta$, IL6 , IL-10, and TNF- $\alpha$. The serum levels of IL-1 $\beta$ and IL-6 were 
higher in the OVX-C group than in the sham group. It is generally regarded that IL-1 and IL- 6 are associated with enhancement of osteoclastic activity ${ }^{4}$. Therefore, estrogen deficiency induced by OVX might be associated with the increases of IL- $1 \beta$ and IL- 6 in serum, and the results indicate that the reduction in bone volume or histomorphometric parameters might be due to elevated osteoclastic activity. The serum level of TNF- $\alpha$ was also increased after OVX. TNF- $\alpha$ is produced by monocytes and macrophages, and it has been implicated in bone resorption by stimulating osteoclast formation ${ }^{14)}$. In this study, estrogen deficiency induced by bilateral OVX caused an increase in the serum level of TNF- $\alpha$, and TNF- $\alpha$ might have induced the increase in bone resorption via stimulation of osteoclast formation. Zhang et al. ${ }^{29)}$ advocated that IL-10 is a potent anti-inflammatory cytokine, and a recent study has indicated that IL-10 can contribute to the maintenance of bone mass through inhibition of osteoclastic bone resorption and induction of osteoblastic bone formation. In the author's study, the serum level of IL-10 was measured during the experimental period; however IL-10 was not detected in the luminex cytokine analysis.

Bone histomorphometric parameters, such as BV/TV, Tb.N. and Tb.Th. in the 4 th lumbar vertebra were significantly decreased during the experimental period in the OVX-C group compared to the sham group on the micro-CT scan. In contrast, Tb.S. was significantly increased after OVX. In the femur, reduction in BV/TV was observed along with a prominent decrease in Tb.Th. Thompson et al. ${ }^{25)}$ investigated the loss of cancellous bone between the proximal tibia and lumbar spine. In their analyses of OVX rats, the number of trabeculae was significantly decreased after surgery, whereas trabecular thickness was only slightly affected in proximal tibiae. On the other hand, in lumbar vertebrae, the number of trabeculae was only slightly affected, but trabecular thinning was significantly decreased. Thus, they concluded that vertebrae lost bone by thinning trabeculae and tibiae lost bone by eliminating trabeculae. However, in the present study in OVX mice, opposite results compared to those in the study by Thompson et al. ${ }^{25)}$ were obtained. The author thought that lumbar spine has a more prominent trabecular bone compared to the long bone, and the trabecular bone in mice has lesser thickness compared to that in rats; therefore, the reduction in Tb.N. was more prominent in the lumbar vertebra compared to the long bone.
Rutin is a kind of bioflavonoid glycoside, and it is found in buckwheat, fruits, vegetables, tea, and wine. Rutin is known as vitamin $\mathrm{P}$ and it has anti-oxidative, anti-inflammatory, anti-viral, anti-carcinogenic, and anti-hypertensive properties $^{11,12)}$. Recent studies investigated the protective effects of rutin on the regulation of bone remodeling. Kyung et al. ${ }^{13)}$ demonstrated that rutin inhibits osteoclast formation by decreasing TNF- $\alpha$ in an in-vitro study. Other studies demonstrated that rutin inhibits the expression of inflammatory cytokines, such as IL-1 $\beta$ and IL-6, which are associated with increased osteoclast activity ${ }^{17)}$. Therefore, the author hypothesized that rutin may have a positive effect in maintaining bone homeostasis in an osteopenic condition by decreasing the osteoclast activity with reduction in the serum levels of inflammatory cytokines and TNF- $\alpha$.

In this study, rutin administration after OVX reduced the serum levels of ALP and CTX-1 at 4 and 8 weeks compared to those in the OVX-C group. However, there was no significant difference in the serum level of osteocalcin between the OVX$\mathrm{C}$ and OVX-R groups, and it was maintained at a higher level compared to that in the sham group during the experimental period. The increase in osteocalcin level in the OVX-R group indicated that rutin was able to enhance bone formation in osteoporosis. Horcajada-Molteni et al. ${ }^{8)}$ also demonstrated that rutin can inhibit OVX-induced osteopenia in rats via decrease in the osteoclast activity and enhancement of osteoblast activity. In the cytokine analysis, the reductions in IL-1 $\beta$, IL-6, and TNF- $\alpha$ were also observed in OVX-R group. The results indicate that rutin administration can reduce the serum levels of IL-1 $\beta$, IL- 6 and TNF- $\alpha$, and the reduction in these cytokines plays an important role in reducing the bone turnover rate in osteoporotic conditions. Kyung et al. ${ }^{13)}$ reported that rutin inhibited osteoclast formation by decreasing reactive oxygen species and TNF- $\alpha$. Histomorphometric analysis showed that BV/TV, Tb.Th., and Tb.N. in the 4th lumbar vertebra were improved at 4 and 8 weeks after surgery in the OVX-R group compared to the OVX-C group. Especially, Tb.Th. was prominently increased at 4 weeks after surgery, and the value was slightly decreased at 8 weeks after OVX. Tb.N. was prominently increased at 8 weeks after OVX. The histomorphometric markers in the femur were also improved after rutin administration, but, the degree of improvement was relatively lower than that in the 4 th lumbar vertebra. As already mentioned, the improvement in bone histomorpho- 
metric values was expected because rutin has an anti-inflammatory effect including reduction in IL-1 $\beta$, IL- 6 , and TNF- $\alpha$, which are regarded as the cytokines associated with the activation of osteoclasts. In the 4th lumbar vertebra of mice in the OVX-R group, Tb.N. was increased more at 8 weeks after surgery and Tb.Th. was decreased at 8 weeks after surgery compared to the values at 4 weeks after surgery. The results suggest that there was formation of new bone tissue, and they indicated that rutin might be associated with activation of osteoblast activity. Continuous administration of rutin might stimulate the osteoblastic activity, and the values of Tb.N. might be increased as a result of increased osteoblastic activity. However, Tb.Th. could be decreased due to the new bony trabecular formation, which might not fully mature during the 8 weeks experimental period. Horcajada-Molteni et al. ${ }^{8)}$ reported that rutin administration can reduce both plasma osteocalcin concentration and urinary deoxypyridinoline excretion. Therefore, they concluded that rutin administration could influence the reduction of osteoclastic activity and enhancement of new bone formation. Although the author could not prove that the rutin administration can affect the induction of osteoblastic activity, histomorphometric results showed some evidence of new bone formation in the OVX-R group.

Although BV/TV of the 4th lumbar vertebra and the femur was significantly decreased at 4 and 8 weeks after OVX, the extent of the decrease was greater in the 4 th lumbar vertebra, which mainly consists of trabecular bone compared to the femur. Ferretti et al. ${ }^{5)}$ reported that bone mass started to decrease earlier and more extensively in trabecular bone than in cortical bone, and the author observed similar findings in the present study. In the 4th lumbar vertebra, BV/TV was decreased at 4 weeks after OVX due to the reduction in Tb.Th. compared to Tb.N. At 8 weeks after OVX, Tb.N. was also significantly decreased in the 4 th lumbar vertebra. However, the reduction in BV/TV and Tb.N. was lesser in the femur compared to the 4th lumbar vertebra. In this study, the percentage of recovery in BV/TV, Tb.Th. and Tb.N. was higher in the 4th lumbar vertebra compared to the femur, and the results indicate that rutin administration might more selectively affect bone recovery and formation of trabecular bone structure. Osteopenia or osteoporosis is initiated in the trabecular bone structure; therefore, rutin could be an effective agent in the patients with early bone loss. Unfortunately, the authors did not perform the biomechanical study to bone strength. To clarify the effect of rutin to osteopenia or osteoporosis, biomechanical study to bone strength will be mandatory.

In the present study, rutin was injected intraperitoneally, and concentration of rutin was $50 \mathrm{mg} / \mathrm{kg} /$ day. HorcajadaMolteni et al. ${ }^{8}$ administered $250 \mathrm{mg} / \mathrm{kg} /$ day of rutin via oral feeding in their rat OVX model. In this study, the author decided to use intraperitoneal injection for rutin administration, and hypothesized that intraperitoneal delivery might be superior compared to oral delivery. Therefore, a relatively low dose of rutin was used compared to that in the study by HorcajadaMolteni et al. ${ }^{8)}$. However, the optimal dose of rutin for successful treatment of osteoporosis still remains to be determined. Especially, the bioavailability of rutin for absorption in an in-vivo study should be firstly ascertained. Unfortunately, the author did not measure the circulating level of rutin or its metabolite. In the future, a study for assessing the adequate rutin concentration and for providing a method to enhance bone health needs to be performed.

\section{CONCLUSION}

The present study demonstrated that rutin therapy has therapeutic effects on improve the bone histomorphometric values and biochemical markers of bone turn-over in OVX mouse model. The improvement of histomorphometric values and biomechanical marker of bone turnover mainly were associated with reduction of osteoclast activity with some evidences of new bone formation. Future, the mechanism of the rutin effect on osteoporosis should be found more clearly to use the rutin on human osteoporosis.

\section{CONFLICTS OF INTEREST}

No potential conflict of interest relevant to this article was reported.

\section{AUTHOR CONTRIBUTIONS}

\author{
Conceptualization : JWJ \\ Data curation : HHL \\ Formal analysis : HHL
}


Funding acquisition : JWJ

Methodology: JKL

Project administration : CKP

Visualization : CKP

Writing - original draft : JWJ

Writing - review \& editing : JKL

\section{ORCID}

Hye-Hwa Lee

https://orcid.org/0000-0002-1061-6023

Jae-Won Jang

https://orcid.org/0000-0001-5555-4359

Jung-Kil Lee

https://orcid.org/0000-0002-9143-4917

Choon-Keun Park https://orcid.org/0000-0003-1566-4726

\section{- Acknowledgements}

Chonnam National University Hospital research institute of clinical medicine provided financial support in the form of a grant (2011-CURIMS-DR012) funding.

\section{References}

1. Adachi JD, Loannidis G, Berger C, Joseph L, Papaioannou A, Pickard L, et al. : The influence of osteoporotic fractures on health-related quality of life in community-dwelling men and women across Canada. Osteoporos Int 12 : 903-908, 2001

2. Burge $R$, Dawson-Hughes $B$, Solomon DH, Wong JB, King A, Tosteson A : Incidence and economic burden of osteoporosis-related fractures in the United States, 2005-2025. J Bone Miner Res 22 : 465-475, 2007

3. Cho DC, Kim KT, Jeon Y, Sung JK : A synergistic bone sparing effect of curcumin and alendronate in ovariectomized rat. Acta Neurochir (Wien) $154:$ 2215-2223, 2012

4. Ersan N, van Ruijven LJ, Bronckers AL, Olgaç V, Ilgüy D, Everts V : Teriparatide and the treatment of bisphosphonate-related osteonecrosis of the jaw: a rat model. Dentomaxillofac Radiol 43 : 20130144, 2014

5. Ferretti M, Bertoni L, Cavani F, Zavatti M, Resca E, Carnevale G, et al. : Influence of ferutinin on bone metabolism in ovariectomized rats. II: role in recovering osteoporosis. J Anat $217:$ 48-56, 2010

6. French $\mathrm{DL}$, Muir JM, Webber CE : The ovariectomized, mature rat model of postmenopausal osteoporosis: an assessment of the bone sparing effects of curcumin. Phytomedicine 15 : 1069-1078, 2008

7. Giuliani N, Pedrazzoni M, Passeri G, Negri G, Impicciatore M, Girasole G : Bisphosphonates stimulate the production of basic fibroblast growth factor and the formation of bone marrow precursors of osteoblasts. New findings about their mechanism of action. Minerva Med 89 :
249-258, 1998

8. Horcajada-Molteni MN, Crespy V, Coxam V, Davicco MJ, Rémésy C, Barlet JP : Rutin inhibits ovariectomy-induced osteopenia in rats. J Bone Miner Res 15 : 2251-2258, 2000

9. Isai M, Sakthivel M, Ramesh E, Thomas PA, Geraldine P : Prevention of selenite-induced cataractogenesis by rutin in Wistar rats. Mol Vis 15 : 2570-2577, 2009

10. Kanis JA, Melton LJ 3rd, Christiansen C, Johnston CC, Khaltaev N : The diagnosis of osteoporosis. J Bone Miner Res 9 : 1137-1141, 1994

11. Kaptoge S, Beck TJ, Reeve J, Stone KL, Hillier TA, Cauley JA, et al. : Prediction of incident hip fracture risk by femur geometry variables measured by hip structural analysis in the study of osteoporotic fractures. J Bone Miner Res 23 : 1892-1904, 2008

12. Korkmaz $A$, Kolankaya $D$ : Protective effect of rutin on the ischemia/ reperfusion induced damage in rat kidney. J Surg Res 164 : 309-315, 2010

13. Kyung TW, Lee JE, Shin HH, Choi HS : Rutin inhibits osteoclast formation by decreasing reactive oxygen species and TNF-alpha by inhibiting activation of NF-kappaB. Exp Mol Med 40 : 52-58, 2008

14. Lam J, Takeshita S, Barker JE, Kanagawa O, Ross FP, Teitelbaum SL : TNF-alpha induces osteoclastogenesis by direct stimulation of macrophages exposed to permissive levels of RANK ligand. J Clin Invest 106 : 14811488,2000

15. Lumachi F, Frigo AC, Basso U, Tombolan V, Ermani M : Estrogen therapy and risk of breast cancer in postmenopausal women: a case-control study and results of a multivariate analysis. Menopause 17 : 524-528, 2010

16. Manach $C$, Morand $C$, Demigné $C$, Texier $O$, Régérat F, Rémésy $C$ : Bioavailability of rutin and quercetin in rats. FEBS Lett $409: 12-16,1997$

17. Middleton ET, Steel SA, Aye M, Doherty SM : The effect of prior bisphosphonate therapy on the subsequent BMD and bone turnover response to strontium ranelate. J Bone Miner Res 25 : 455-462, 2010

18. Omi N, Ezawa I: The effect of ovariectomy on bone metabolism in rats. Bone 17 : 163S-168S, 1995

19. Pabon M, Tamboli C, Tamboli S, Acosta S, De La Pena I, Sanberg PR, et al. : Estrogen replacement therapy for stroke. Cell Med 6 : 111-122, 2014

20. Palumbo C, Ferretti M, Bertoni L, Cavani F, Resca E, Casolari B, et al. : Influence of ferutinin on bone metabolism in ovariectomized rats. I: role in preventing osteoporosis. J Bone Miner Metab 27 : 538-545, 2009

21. Pazianas M, Abrahamsen B : Safety of bisphosphonates. Bone 49 : 103-110, 2011

22. Reszka AA, Rodan GA : Mechanism of action of bisphosphonates. Curr Osteoporos Rep $1:$ 45-52, 2003

23. Shin YH, Cho DC, Yu SH, Kim KT, Cho HJ, Sung JK : Histomorphometric analysis of the spine and femur in ovariectomized rats using micro-computed tomographic scan. J Korean Neurosurg Soc 52 : 1-6, 2012

24. Songpatanasilp T, Mumtaz M, Chhabra H, Yu M, Sorsaburu S : Back pain in patients with severe osteoporosis on teriparatide or antiresorptives: a prospective observational study in a multiethnic population. Singapore Med J 55 : 493-501, 2014 
Positive Effect of Rutin to Osteoporosis | Lee HH, et al.

25. Thompson DD, Simmons HA, Pirie CM, Ke HZ : FDA guidelines and animal models for osteoporosis. Bone 17 : 125S-133S, 1995

26. Watts NB, Nolan JC, Brennan JJ, Yang HM; ESTRATAB/Osteoporosis Study Group : Esterified estrogen therapy in postmenopausal women. Relationships of bone marker changes and plasma estradiol to BMD changes: a two-year study. Menopause $7:$ 375-382, 2000

27. Weber $M$, Uehlinger $K$, Gerber $H$ : Osteoporotic vertebral compression fracture causing neurologic deficit. J Clin Rheumatol 8 : 166-173,
2002

28. Yang MW, Wang TH, Yan PP, Chu LW, Yu J, Gao ZD, et al. : Curcumin improves bone microarchitecture and enhances mineral density in APP/ PS1 transgenic mice. Phytomedicine 18 : 205-213, 2011

29. Zhang Q, Chen B, Yan F, Guo J, Zhu X, Ma S, et al. : Interleukin-10 inhibits bone resorption: a potential therapeutic strategy in periodontitis and other bone loss diseases. Biomed Res Int 2014 : 284836, 2014 